\title{
Incorporating information quality criteria in blog search engine
}

\begin{abstract}
Weblog and some new social media are cause of an evolution in World Wide Web. Weblogs are web pages with several dated entries usually arranged in reverse chronological order[1]. This form of online diary media has become an influential web application, with thousands of blogs added on the web every day. In general, blog sites cover a wide range of topics. Sites with topics referred to politics or technology-related usually receive thousands of hits per day. In the other hand the amount of information on the web and the number of new user, those are inexperienced at web searching are growing rapidly. These reasons show the importance of search engine on Weblog, so we need to search engine that retrieve high quality and relevance page. This paper will be explained some methods that consider quality and relevancy in search engine.
\end{abstract}

Keyword: Quality; Relevancy; Search engine; Weblog 